\title{
Benevolent viruses in skin cancer
}

control
MmuPV1-
colonized mice
developed
fewer UVB-
induced
tumours than
the CD8
T cell-depleted
MmuPV1-
colonized mice

The risk of cutaneous squamous cell carcinoma (SCC) is increased in patients with immunosuppression and associated with beta human papilloma virus ( $\beta$-HPV). Strickley, Messerschmidt et al. now show that $\beta$-HPV infection itself is not causal in SCC development in the context of immunosuppression, but instead it is the loss of $\beta$-HPV-mediated $\mathrm{T}$ cell immunity that promotes SCC in this context.

$\beta$-HPV infection has been hypothesized to facilitate the initiation of carcinogen-driven skin cancer. The authors used back-skin infection of mouse papillomavirus type 1 (MmuPV1) in mice to model carcinogen-driven SCC. C57BL/6J mice infected with MmuPV1 or sham infected were exposed to a chemical carcinogen protocol 2 months after infection, for 30 weeks. MmuPV1infected mice developed skin tumours, although onset was delayed and the tumour burden was lower than in sham-infected mice. When immunodeficient $\mathrm{Cd} 4^{-/-} \mathrm{Cd} 8^{-/-}$ mice were infected with MmuPV1, they developed confluent warts, in contrast to immunocompetent MmuPV1-infected control mice.

However, when memory $\mathrm{T}$ cells from MmuPV1-infected

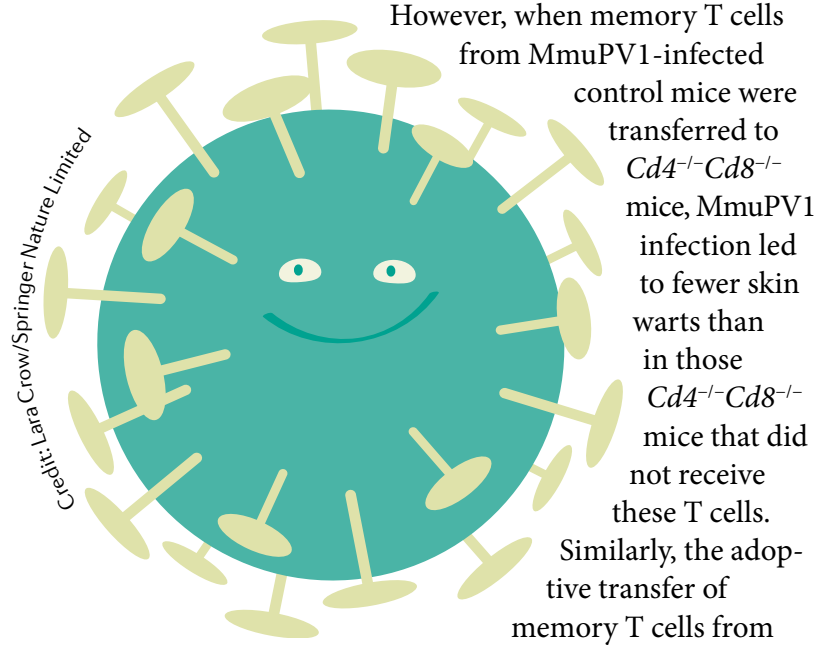

MmuPV1-immune mice led to regression of persistent warts in MmuPV1-colonized FVB mice. These mice were also protected from chemical carcinogenesis, suggesting that T cells from MmuPV1-immune mice confer antiviral adaptive immunity that can protect from carcinogenesis.

Moving on to explore ultraviolet (UV) radiation-induced SCC, the authors used the MmuPV1back-skin infection system in SKH-1 hairless mice. MmuPV1- or sham-infected mice underwent UVB radiation three times a week for 25 weeks, which led to tumour development in both conditions, although MmuPV1-infected mice had fewer tumours and a lower tumour burden than sham-infected mice. Of note, when UVB treatment cycles were completed, the skin of MmuPV1-colonized mice as well as their skin tumours had an increased number of $\mathrm{CD}^{+} \mathrm{T}$ cells compared with sham-infected control mice. When SKH-1 mice underwent antibody-mediated $\mathrm{CD}^{+} \mathrm{T}$ cell depletion after infection with MmuPV1 or sham infection, control MmuPV1-colonized mice developed fewer UVB-induced tumours than the CD8 ${ }^{+} \mathrm{T}$ celldepleted MmuPV1-colonized mice, and fewer tumours than sham(VLP)-infected mice in either treatment group.

Moving on to patients, the authors analysed $\beta$-HPV infection in SCC lesions from patients with or without immunosuppression, using RNA in situ hybridization to detect E6 transcripts of 25 types of $\beta$-HPV. SCC lesions from patients with immunosuppression had higher levels of $\beta$-HPV RNA expression than SCC lesions from immunocompetent patients. Also, $\beta$-HPV RNA expression was reduced in cancer cells compared with adjacent normal skin cells in both groups. These results were reflected in a higher viral load in SCC lesions in patients with immunosuppression compared with immunocompetent patients, and also correlated with fewer tumour- and skin-infiltrating $\mathrm{CD}^{+} \mathrm{T}$ cells in patients with immunosuppression. In line with antiviral adaptive immunity in immunocompetent patients, $\beta$-HPV E7 peptides were able to activate $\mathrm{CD} 8^{+} \mathrm{T}$ cells isolated from the normal facial skin of these patients. Returning to their animal model, the authors performed RNA sequencing of skin warts, MmuPV1-infected UVB-treated skin and tumours, and sham-infected tumours of SKH-1 mice. Among the genes that were upregulated in MmuPV1induced warts and UVB-induced tumours from MmuPV1-infected compared with sham-infected mice, immune-related genes including damage-associated molecular pattern (DAMP) genes were detected.

This study highlights the role of commensal HPVs in priming adaptive immunity in immunocompetent hosts and presents an opportunity for developing antiviral $\mathrm{T}$ cell-based vaccines to prevent skin carcinogenesis.

Ulrike Harjes

ORIGINAL ARTICLE Strickley, J. D. et al. Immunity to commensal papillomaviruses protects against skin cancer. Nature https://doi.org/10.1038/ s41586-019-1719-9 (2019) RELATED ARTICLE Elinav, E. et al. The cancer microbiome. Nat. Rev. Cancer 19, 371-376 (2019) 